University of Nebraska - Lincoln

DigitalCommons@University of Nebraska - Lincoln

$2-2009$

\title{
Kinematic analysis of articulatory coupling in acquired apraxia of speech post-stroke
}

\author{
Carly J. Bartle-Meyer \\ University of Queensland, Brisbane,, c.bartle@uq.edu.au \\ Justine V. Goozée \\ University of Queensland, Brisbane, \\ Bruce E. Murdoch \\ University of Queensland, Brisbane, \\ Jordan R. Green \\ University of Nebraska-Lincoln, jgreen4@unl.edu
}

Follow this and additional works at: https://digitalcommons.unl.edu/specedfacpub

Part of the Special Education and Teaching Commons

Bartle-Meyer, Carly J.; Goozée, Justine V.; Murdoch, Bruce E.; and Green, Jordan R., "Kinematic analysis of articulatory coupling in acquired apraxia of speech post-stroke" (2009). Special Education and Communication Disorders Faculty Publications. 51.

https://digitalcommons.unl.edu/specedfacpub/51

This Article is brought to you for free and open access by the Department of Special Education and Communication Disorders at DigitalCommons@University of Nebraska - Lincoln. It has been accepted for inclusion in Special Education and Communication Disorders Faculty Publications by an authorized administrator of DigitalCommons@University of Nebraska - Lincoln. 


\title{
Kinematic analysis of articulatory coupling in acquired apraxia of speech post-stroke
}

\author{
Carly J. Bartle-Meyer, ${ }^{1}$ Justine V. Goozée, ${ }^{1}$ Bruce E. Murdoch, ${ }^{1}$ and Jordan R. Green ${ }^{2}$ \\ 1. School of Health and Rehabilitation Sciences, The University of Queensland, Brisbane, Queensland, Australia \\ 2. Department of Special Education and Communication Disorders, University of Nebraska-Lincoln, USA \\ Corresponding author - C. J. Bartle-Meyer, e-mail c.bartle@uq.edu.au
}

\begin{abstract}
Primary objective: Electromagnetic articulography was employed to investigate the strength of articulatory coupling and hence the degree of functional movement independence between individual articulators in apraxia of speech (AOS). Methods and procedures: Tongue-tip, tongue-back and jaw movement was recorded from five speakers with AOS and a concomitant aphasia (M $=53.6$ years; $\mathrm{SD}=12.60)$ during $/ \mathrm{ta}, \mathrm{sa}, \mathrm{la}, \mathrm{ka} /$ syllable repetitions, spoken at typical and fastrates of speech. Covariance values were calculated for each articulatory pair to gauge the strength of articulatory coupling. The results obtained for each of the participants with AOS were individually compared to those obtained by a control group $(n=12 ; M=52.08$ years; SD = 12.52$)$. Comparisons were made between the typical rate productions of the control group and the typical and fast rate productions of the participants with AOS.

Main outcomes and results: In comparison to the control group, four speakers with AOS exhibited significantly stronger articulatory coupling for alveolar and/or velar speech targets, during typical and/or fast rate conditions, suggesting decreased functional movement independence.

Conclusions: The reduction in functional movement independence might have reflected an attempt to simplify articulatory control or a decrease in the ability to differentially control distinct articulatory regions.
\end{abstract}

Keywords: Apraxia of speech, electromagnetic articulography, articulation, coupling

\section{Introduction}

Acquired apraxia of speech (AOS) is a neurogenic speech disorder that most commonly occurs following single left-hemisphere stroke [1]. Disturbances in prosody and articulation, caused by an underlying motor planning/programming impairment, are typical of AOS. These disturbances have been found to manifest as sound distortions, distorted sound substitutions, sound prolongation, slow, syllabic speech output and dysprosody [1-5].

In an attempt to determine the underlying physiological nature of the aforementioned articulatory dis- turbances, investigations have studied lip and tongue movements during speech production. Results have indicated that lower lip (plus jaw) movement in AOS is characterized by prolonged movement durations [6-8]. Researchers have also reported that movements of the lower lip (plus jaw) are significantly larger [6, 8], slower $[9,10]$ and more variable [10] in persons with AOS, compared to healthy talkers; however, in other studies, no systematic differences in displacement [7] and peak velocity $[6-8,11]$ have been observed between participants with AOS and healthy talkers.

Similar results have been reported from investigations of lingual kinematics in AOS. Namely, significantly 
prolonged approach, closure and/or release phase durations and, in some instances, significantly greater articulatory displacements have been reported [12-14]. Bartle et al. [12] reported significant reductions in maximum acceleration, maximum deceleration and maximum velocity. However, more recent investigations of tongue movement in AOS have revealed relatively unimpaired measures of lingual speed $[13,14]$. Nevertheless, the majority of the aforementioned studies suggest that AOS is associated with tongue and lip movement abnormalities during speech.

Having established that participants with AOS evidence deviant labial and lingual kinematics during speech production, it could be asserted that this population would also exhibit difficulties with coordinated articulatory movement. In accordance with task dynamics, articulate speech is achieved through the activation of dynamically-defined articulatory gestures [15]. Importantly, these articulatory gestures allow a remarkable degree of task-specific coordinative flexibility between articulators, for example, between the lips and jaw and the tongue and jaw, during speech production [15-19]. Indeed, Mooshammer et al.'s [19] research findings indicated that different coronal constrictions could be achieved through variable jaw contributions; the fricatives /s, s/, and the voiceless plosive / $t$ / were achieved with a high jaw position, whereas the voiced coronals / $\mathrm{d}, \mathrm{n}, \mathrm{l} / \mathrm{were}$ achieved with a low jaw position.

Similarly, a recent kinematic investigation of lingual coupling during typical speech production revealed a high degree of functional movement independence across four lingual regions (i.e. anterior blade, posterior blade, tongue body and tongue dorsum) [20]. The coupling profiles generated were sufficiently able to differentiate consonants based on their place of articulation, with the strength of coupling being greatest between contiguous lingual regions near the primary place of closure/constriction[20]. Specifically, the strength of lingual coupling was strongest between the anterior and posterior tongue blades during alveolar, retroflex and postalveolar consonants and between the tongue body and tongue dorsum and, to a lesser extent, posterior blade and tongue body, during velar consonants [20]. Significantly stronger negative coupling (i.e. tongue front raising and simultaneous tongue back lowering) between the anterior blade and tongue dorsum was evident during alveolar, retroflex and post-alveolar consonants, relative to other lingual pairs (with the exception of posterior blade and tongue dorsum coupling); however, all lingual pairs were positively coupled during more posterior consonants [20]. With one exception, the lateral approximant /1/ was consistently characterized by low coupling between distinct lingual regions; the anterior blade and tongue body demonstrated significantly stronger negative coupling [20].

Evidence of limited functional movement independence has been observed in AOS. Frequent double alveolar-velar contacts were exhibited for alveolar and velar speech targets by two of four participants with AOS in an electropalatographic (EPG) investigation of lingual movement in participants with AOS [21]. Other EPG investigations of articulation in participants with AOS have also reported double articulation patterns [22-26]. A double alveolar-velar pattern could be indicative of a single, undifferentiated lingual gesture up to the palate, which could indicate that participants with AOS are less able to exert independent control over quasi-independent articulatory structures. No research to date, however, has investigated the strength of articulatory coupling in participants with AOS to verify this claim.

Speech rate has had a documented impact on articulatory kinematics. Goozée et al. [27] examined lingual movement during a syllable repetition task wherein a group of eight healthy talkers were instructed to repeat a set of syllables at a moderate (i.e. three syllables per second) and fast speech rate (i.e. as fast as possible). Results indicated that $87.5 \%$ of the participants achieved a faster rate of speech by reducing the total distance travelled by the tongue; two of these participants also evidenced a reduction in maximum velocity and one participant evidenced an increase in maximum velocity and subsequently maximum acceleration [27]. In a follow-up study, Goozée et al. [28] reported that both younger and older adults appear to increase syllable repetition rate by reducing the distance travelled by the tongue. The impact of rate on jaw and constrictor (i.e. lip, tongue) kinematics was examined by Hertrich and Ackermann [29]. In accordance with the notion of functional movement independence, Hertrich and Ackermann [29] discovered that the jaw and constrictor articulators were differentially affected by changes in speech rate. Where jaw amplitude appeared to be relatively insensitive to changes in speech rate, lower-lip movement tended to decrease in amplitude as syllable repetition rate increased. On the contrary, where the constrictors appeared to be relatively insensitive to rate changes with respect to velocity/amplitude scaling (i.e. stiffness), the jaw demonstrated a relatively proportional change of stiffness with an increase in speech rate [29]. No research to the authors' knowledge has examined the impact of speech rate on the strength of articulatory coupling; however, given that rate of speech undoubtedly influences lip, tongue and jaw kinematics, it would appear paramount that investigations of articulatory coupling in neurogenic populations control for speech rate. 


\begin{tabular}{lcccc}
\multicolumn{5}{l}{ Table I. Medical and biographical details for each participant with apraxia of speech (A1-A5). } \\
\hline Participant & Age (years) & Gender & Time post-stroke (years) & Neuropathology \\
\hline A1 & 35 & F & $1 ; 10$ & $\begin{array}{c}\text { Left MCA ischaemic CVA secondary to internal carotid artery } \\
\text { dissection; parietal infarct } \\
\text { Not detected by CT scan }\end{array}$ \\
A3 & 63 & F & $2 ; 9$ & $\begin{array}{c}\text { Bilateral ischaemic infarction in the left fronto-parietal and } \\
\text { right posterior parietal regions }\end{array}$ \\
A4 & 54 & M & 1 & $\begin{array}{c}\text { Haemorrhagic CVA; Left frontal infarct } \\
\text { Left MCA ischaemic CVA; internal cerebral artery occlusion }\end{array}$ \\
A5 & 67 & M & $1 ; 9$ & 1
\end{tabular}

Note: $\mathrm{F}$ = female; $\mathrm{M}=$ male; $\mathrm{MCA}$ = middle cerebral artery; $\mathrm{CVA}$ = cerebrovascular accident.

The present study aimed to use electromagnetic articulography (EMA) to investigate the strength of spatiotemporal coupling and thus the degree of functional movement independence between individual articulators during a syllable repetition task. Given that participants with AOS typically speak at a reduced rate of speech [2], participants were asked to produce the syllables at a typical and fast rate of speech in an attempt to eliminate the impact of speech rate on the strength of spatiotemporal coupling. It was hypothesized that the participants with AOS would exhibit increased spatiotemporal coupling between individual articulators, to reflect a reduction in functional movement independence. An increase in speech rate was anticipated to result in weaker coupling, given that a fast rate of speech can be associated with a reduction in articulatory displacement [27-29].

\section{Method}

\section{Participants}

Five participants (A1-A5) (two male, three female; age range $=35-67$ years; $\mathrm{M}=53.6$ years; $\mathrm{SD}=12.60$ ) who presented with AOS and a concomitant non-fluent aphasia participated in the study; each of their medical and biographical details is described in Table I. All participants were a minimum of 12 months post-onset of a stroke $(\mathrm{M}=1.67$ years; $\mathrm{SD}=0.72)$ at the time of assessment. Three of the participants with AOS were diagnosed with a single left-hemisphere stroke and one participant was diagnosed with a bilateral stroke. A2's CT scan failed to detect an infarct; however, clinical signs (e.g. right side weakness, dysphasia) were suggestive of a left hemisphere stroke and therefore she was included in the study. Participants were excluded if they presented with a history of speech or neurological disorder unrelated to their stroke or had previously undergone oro-maxillofacial surgery.

To determine each participant's speech pathology diagnosis at the time of assessment, the Apraxia Battery for Adults-Second Edition (sections 1-5; ABA-2) [30], the Boston Diagnostic Aphasia Examination-Third Edition (Short Form; BDAE-3) [31] and the Frenchay Dysarthria Assessment (tongue section only; FDA) [32] were administered. Each participant's assessment results are presented in Table II. The participants with AOS were also required to provide a spontaneous speech sample and were instructed to read aloud 'The Grandfather Passage' [33] at a habitual rate and loudness level. An independent speech pathologist screened each participant's 'Grandfather Passage' reading and spontaneous speech sample, to confirm the diagnosis of AOS and to judge the severity of mandatory characteristics (i.e. sound distortions, sound prolongations, syllabic speech output and dysprosody) [2]. The principal researcher and an independent speech pathologist agreed upon overall severity of AOS. In each case, AOS was judged to be the primary communication disorder.

As described in previous investigations conducted by the principal researcher [13, 14], A1 was diagnosed with a moderate-severe AOS with a mild oral apraxia. A1 was rated as having no impairment in sub-test $2 \mathrm{~A}$ (increasing word length-1) of the ABA-2; however, this was due to A1's significant difficulties with mono- and multisyllabic words. Because of A1's substantial difficulties with oral expression, $\mathrm{A} 1$ did not complete sub-test $2 \mathrm{~B}$ (increasing word length-2) or 5 (repeated trials) of the ABA-2. A1's speech was characterized by frequent sound distortions, moderate sound prolongations, a high frequency of intersyllabic pauses (i.e. syllabic speech) and a severe reduction in the temporal flow of speech (i.e. dysprosody) (NB: A1 was unable to read aloud "The Grandfather Passage" and therefore the aforementioned judgements were based on her spontaneous speech sample only). On the BDAE-3, A1 was diagnosed with a non-fluent aphasia. A1 scored poorly on the Basic Word Discrimination sub-test of the BDAE-3; however, this was largely due to significant delays in response time, attributable to AOS. The FDA revealed that A1's tongue did deviate noticeably to the right at rest; however, she exhibited unimpaired lingual protrusion, tongue elevation and lateral tongue movement. 


\begin{tabular}{|c|c|c|c|c|c|c|}
\hline \multicolumn{7}{|c|}{ Apraxia Battery for Adults (2nd ed.): } \\
\hline \multicolumn{2}{|c|}{ 2A. Increasing word length (1) } & None & Mild & Mild & None & Mild \\
\hline \multicolumn{2}{|c|}{ 2B. Increasing word length (2) } & - & Severe & Severe & Moderate & Severe \\
\hline \multicolumn{2}{|l|}{ 3A. Limb apraxia } & None & None & None & None & None \\
\hline \multicolumn{2}{|c|}{ 5. Repeated trials } & - & Mild & Moderate & Mild & Moderate \\
\hline \multicolumn{7}{|c|}{ Boston Diagnostic Aphasia Examination (3rd ed.): } \\
\hline \multicolumn{2}{|l|}{ Severity rating } & 1 & 3 & 2 & 2 & 2 \\
\hline \multirow[t]{2}{*}{ Fluency } & Phrase length & $3 / 7$ & $7 / 7$ & $4 / 7$ & $5 / 7$ & $5 / 7$ \\
\hline & Melodic line & $1 / 7$ & $4 / 7$ & $3 / 7$ & $2 / 7$ & $3 / 7$ \\
\hline Articulation & Articulatory agility & $2 / 7$ & $4 / 7$ & $3 / 7$ & $3 / 7$ & $4 / 7$ \\
\hline \multirow{3}{*}{$\begin{array}{l}\text { Recitation } \\
\text { Repetition }\end{array}$} & Automatized sequences & $1 / 4$ & $4 / 4$ & $4 / 4$ & $4 / 4$ & $4 / 4$ \\
\hline & Words & $2 / 5$ & $4 / 5$ & $5 / 5$ & $4 / 5$ & $4 / 5$ \\
\hline & Sentences & $0 / 2$ & $1 / 2$ & $2 / 2$ & $2 / 2$ & $1 / 2$ \\
\hline \multirow[t]{3}{*}{ Naming } & Responsive naming & $4 / 10$ & $10 / 10$ & $10 / 10$ & $8 / 10$ & $8 / 10$ \\
\hline & Boston naming test & $0 / 15$ & $10 / 15$ & $15 / 15$ & $9 / 15$ & $15 / 15$ \\
\hline & Special categories & $2 / 12$ & $11 / 12$ & $12 / 12$ & $12 / 12$ & $12 / 12$ \\
\hline \multicolumn{7}{|c|}{ Frenchay Dysarthria Assessment (tongue): } \\
\hline \multicolumn{2}{|l|}{ At rest } & $\mathrm{C}$ & B & $\mathrm{B}$ & $\mathrm{B}-\mathrm{C}$ & $\mathrm{B}-\mathrm{C}$ \\
\hline \multicolumn{2}{|l|}{ Protrusion } & A & A & A & B & A \\
\hline \multicolumn{2}{|l|}{ Elevation } & A & $\mathrm{D}$ & $\mathrm{C}$ & $\mathrm{C}$ & A \\
\hline \multicolumn{2}{|l|}{ Lateral } & A-B & A-B & $\mathrm{E}$ & A & A \\
\hline
\end{tabular}

Note: ' $\boldsymbol{-}^{\prime}=$ inability to complete sub-test; $\mathrm{A}=$ no impairment, $\mathrm{B}=$ mild impairment, $\mathrm{C}=$ moderate impairment, $\mathrm{D}=$ severe impairment, $\mathrm{E}=$ profound impairment.

A1's alternate tongue movement was severely impaired during speech (e.g. / ka la/) and this was most likely the result of AOS.

A2 was diagnosed with a moderate AOS and no oral apraxia. She exhibited a moderate number of sound distortions, mild-to-moderate sound prolongations, frequent inter-syllabic pauses (i.e. syllabic speech) and a moderate-to-severe reduction in the temporal flow of speech (i.e. dysprosody). A2's BDAE-3 profile indicated a non-fluent aphasia. On the FDA, A2 evidenced minimal tongue deviation to the right at rest. No or minimal disturbances with tongue protrusion and lateral tongue movement were identified during the FDA. However, A2 did exhibit a severe disturbance with tongue elevation and a mild-moderate disturbance with alternate tongue movement during speech. Both these disturbances were attributable to AOS.

A3 was diagnosed with a mild-moderate AOS and no oral apraxia. He exhibited occasional sound distortions, moderate sound prolongations, a moderate number of inter-syllabic pauses (i.e. syllabic speech) and a moderate reduction in the temporal flow of speech (i.e. dysprosody). A3 was diagnosed with minimal, non-fluent aphasia on the BDAE-3. His FDA profile indicated minimal tongue deviation to the right at rest and normal tongue protrusion ability. Moderate disturbances in tongue elevation, profound disturbances in lateral tongue movement and severe disturbances in alternate tongue movement during speech, however, were exhibited by A3 during the tongue section of the FDA. Significant difficulties with coordinated tongue movement, as a result of AOS, presumably impacted on A3's ability to perform these latter tasks.

A4 was diagnosed with a moderate AOS and a mild oral apraxia. He exhibited frequent sound distortions, mild-to-moderate sound prolongations, frequent intersyllabic pauses (i.e. syllabic speech) and a moderateto-severe reduction in the temporal flow of speech (i.e. dysprosody). A4's performance on the BDAE-3 was indicative of a non-fluent aphasia. On the FDA, A4 exhibited minimal tongue deviation to the right at rest. However, A4 evidenced either no or only mild difficul- 
ties with tongue protrusion and lateral tongue movement. Tongue elevation and alternate tongue movement during speech were both moderately impaired, both of which were attributed to AOS.

A5 was diagnosed with a mild AOS and no oral apraxia. She exhibited occasional sound distortions, mild sound prolongations, occasional intersyllabic pauses (i.e. syllabic speech) and a mild reduction in the temporal flow of speech (i.e. dysprosody). A5's BDAE-3 results indicated minimal, non-fluent aphasia. On the FDA, A5 presented with noticeable tongue deviation to the right. However, she exhibited unimpaired lingual movement during the tongue protrusion, tongue elevation and lateral tongue movement tasks. A5 exhibited mild-to-moderate difficulties with alternate tongue movement during speech, which was thought to reflect AOS.

A control group of eight male and four female nonneurologically impaired participants (age range $=29$ 70 years; $\mathrm{M}=52.08 ; \mathrm{SD}=12.52$ ) also participated in the study. Participants were all native English speakers and did not present with a history of or any form of speech and/or neurological disorder or any history of lip, tongue or jaw surgery.

\section{Assessment procedures}

Articulatory movement along the mid-sagittal plane was recorded during speech production using the Electromagnetic Articulograph AG200 system (Carstens Medizinelektronik GmbH, Germany). Similar methodology to that employed in past EMA investigations conducted within the Motor Speech Research Centre was employed in the present study [13, 14, 34].

\section{Physiological assessment}

Instrumentation. A plastic helmet comprising three transmitter coils was secured to the participants' heads. Each of the transmitter coils (positioned in front of the participants' jaw, behind the neck and in front of the forehead within the mid-sagittal plane) produced an alternating magnetic field at different frequencies (range $10-20 \mathrm{kHz}$ ), which induced alternating signals in seven receiver coils $(\sim 2 \times 2 \times 3 \mathrm{~mm}$ in size $)$ that were attached to the participants' tongue, lips and jaw within the midline. The position ( $x-y$ coordinates) of the receiver coil within the two-dimensional mid-sagittal plane was calculated based on the magnitude of the signals induced in the receiver coil (magnitude of signal is inversely proportional to cube of distance from the transmitter coil). All position data was sampled at a measurement frequency of $200 \mathrm{~Hz}$.
Assessment preparation. The AG200 system was calibrated according to the procedures outlined in the AG200 operating manual. Receiver coils were affixed to the participants' tongue-tip $(1 \mathrm{~cm}$ from the tip of the tongue), tongue-back ( $4 \mathrm{~cm}$ from the tongue-tip), upper and lower lips and under the mental protuberance of the mandible, each within the midline. Receiver coils were also attached to the bridge of the nose and to the gingiva above the two upper central incisors to enable correction for any head movement that may have occurred in relation to the helmet. The receiver coils were coated in latex (plasty-late) and attached to the articulators using a biologically safe adhesive (Cyano-Vaneer Fast, a cyanoethyl liquid); tape was used to attach the receiver coils to the nose and jaw. The plastic helmet containing the transmitter coils was secured to the participants' head once all but one of the receiver coils were attached; the unattached receiver coil was positioned in the T-junction at the end of a custom made T-bar to trace the occlusional plane. To obtain the trace, the researcher ran ends of the t-bar over the participants' upper teeth, from the molars to the first or second bicuspid, with the receiver coil in the midline. The receiver coil was subsequently attached to the participants' tongue-tip.

Recording procedure. Consistent with prior EMA investigations [34], the participants were allowed _5 minutes of speaking time prior to the assessment to become accustomed to speaking with the receiver coils on the articulators. The EMA assessment began once the principal researcher judged the participants' speech to be unaffected by coil placement. The participants were subsequently instructed to repeat after the researcher a set of syllables, which were modelled at a rate of three syllables per second for the typical rate condition and at five syllables per second for the fast rate condition. Each syllable adhered to a CV (i.e. / ta, sa, la, ka/) construction and was repeated 12 times in a train, twice at a typical speaking rate and twice at a fast speaking rate. While the participants repeated the syllables, their tonguetip, tongue-back and jaw movements were recorded. A lapel microphone that was connected to the AG200 EMA system was attached to the front of the participants' helmet to record the acoustic signals (sampling rate $1 / 416$ $\mathrm{kHz}$ ) during the EMA assessment. The preparation and recording procedures took $\sim 60$ minutes for each participant.

\section{Analysis procedures}

Data preparation. The Tailor programme (Carstens Medizinelektronik $\mathrm{GmbH}$, Germany) was used to modify the kinematic data recorded by the AG200 sys- 
tem in preparation for data analysis. The reference (Filter 40 , cut-off $=8 \mathrm{~Hz}$ ) and data (Filter 160, cut-off $=$ $32 \mathrm{~Hz}$ ) channels were filtered initially, after which dynamic correction took place to correct for any head movement. Finally, the movement data was rotated within the $x-y$ coordinate system to ensure that the occlusional plane was parallel to the $x$-axis and that the orientation of kinematic data was consistent between the participants.

Movements recorded by the tongue receiver coils represented the combined motions of jaw and tongue. Obtaining tongue motion that was independent from jaw motion was not necessary to address the primary goal of the current study, which was to identify differences in articulatory performance between the participants with AOS and healthy controls. Moreover, preserving the natural coupling between jaw and tongue movements provided a way to determine the functional significance of increased articulatory coupling on overall vocal tract configuration.

Analysis of articulatory coupling. The tongue-tip (/ta, $\mathrm{sa}, \mathrm{la} /)$ and tongue-back (/ka/) y-displacement and tangential velocity profiles produced by Emalyse (Carstens Medizinelektronik $\mathrm{GmbH}$, Germany) during the syllable repetition task were used to parse the syllable data prior to analysis. A string of eight consecutive syllables was typically isolated for analysis of articulatory coupling; however, in less than $2 \%$ of cases, syllable trains of five-to-seven syllables were accepted. The minimum velocity point at the onset of the approach phase (i.e. the tongue approaching the palate) of the first syllable within the string of syllables defined the start-point and the minimum velocity point at the end of the release phase (i.e. the tongue moving downward from the palate) of the final syllable within the string of syllables defined the end-point (see Figure 1). Syllable data was excluded from the analysis if the receiver coil affixed to the tongue and/or jaw malfunctioned or if the participant consistently substituted a non-target sound for a target sound within the string of syllables. Consequently, $\sim 11 \%$ of the syllable data was not included in analysis. In some cases, participants were unable to produce the syllable sequence a second time.

Custom written scripts in Matlab_ (r2006b, version 7.3.0) were used to investigate the strength of spatiotemporal coupling between the tongue and jaw (i.e. TT $\times \mathrm{J}$, $\mathrm{TB} \times \mathrm{J}$ ) and tongue-tip and tongueback (i.e. TT $\times \mathrm{TB}$ ) during / ta, sa, la, ka/ repetitions (see Figure 2). The $y$-position signals were initially smoothed using a low pass filter $\left(f_{1 \mathrm{p}}=15 \mathrm{~Hz}\right)$. Subsequently, covariance values were computed on the filtered signals for the $\mathrm{TT} \times \mathrm{J}, \mathrm{TB} \times \mathrm{J}$ and $\mathrm{TT} \times \mathrm{TB}$ receiver coil pairs (see Figure 3 ). As described

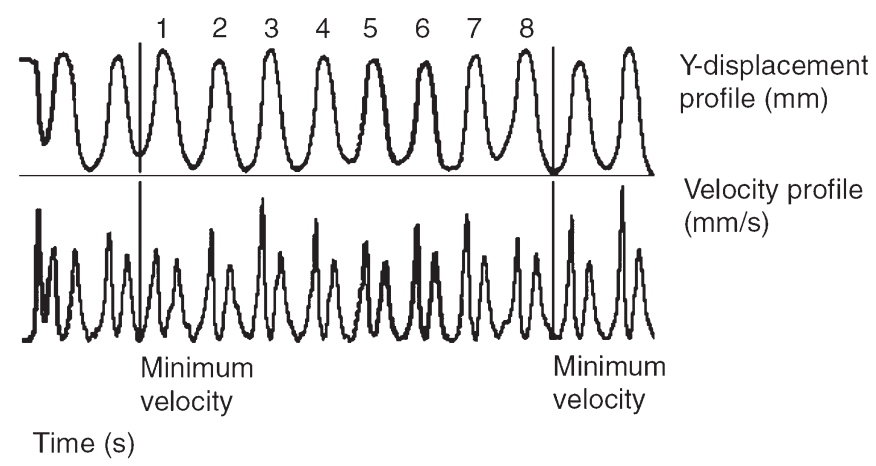

Figure 1. Example $y$-displacement and velocity profiles for the tongue-tip during / ta/ syllable repetitions, which were used to parse the syllable data prior to analysis. Eight consecutive syllables were isolated for analysis. The minimum velocity point at the start of the approach of the first syllable within the eight-syllable string defined the start-point and the minimum velocity point at the end of the release of the last syllable within the eight-syllable string defined the end-point.

by Green and Wang [20] and as displayed in equation (1), covariance (Cov) reflects the zero-lag correlation $(r)$ between the vertical-time histories of two receiver coils, as well as their associated standard deviations (SD). Therefore, covariance represents the strength of correlation between the movements of two articulators that is weighted by the amplitude of their individual movements.

$$
\mathrm{Cov}_{x y}=r_{i j} \times \mathrm{SD}_{i} \times \mathrm{SD}_{j}
$$

A high degree of spatiotemporal coupling/low degree of functional movement independence was inferred from a high covariance value; limited spatiotemporal coupling/a high degree of functional movement independence was inferred from a low covariance value. For each rate condition, an average covariance value was calculated across the two syllable repetitions.

The CV utterances were expected to yield predominantly positive covariance values as the tongue and jaw move synchronously during oral closure for the consonant and during oral opening for the vowel. Low, positive covariance values were anticipated if one of the articulators produced most of the movement during consonant closure with minimal assistance from the other. In contrast, high, positive covariance values were expected if both articulators contributed significantly to the gesture. It was hypothesized that the participants with AOS would exhibit relatively larger covariance values than healthy controls because of a decrease in the independence of movement between the tongue and jaw and between the anterior and posterior regions of 

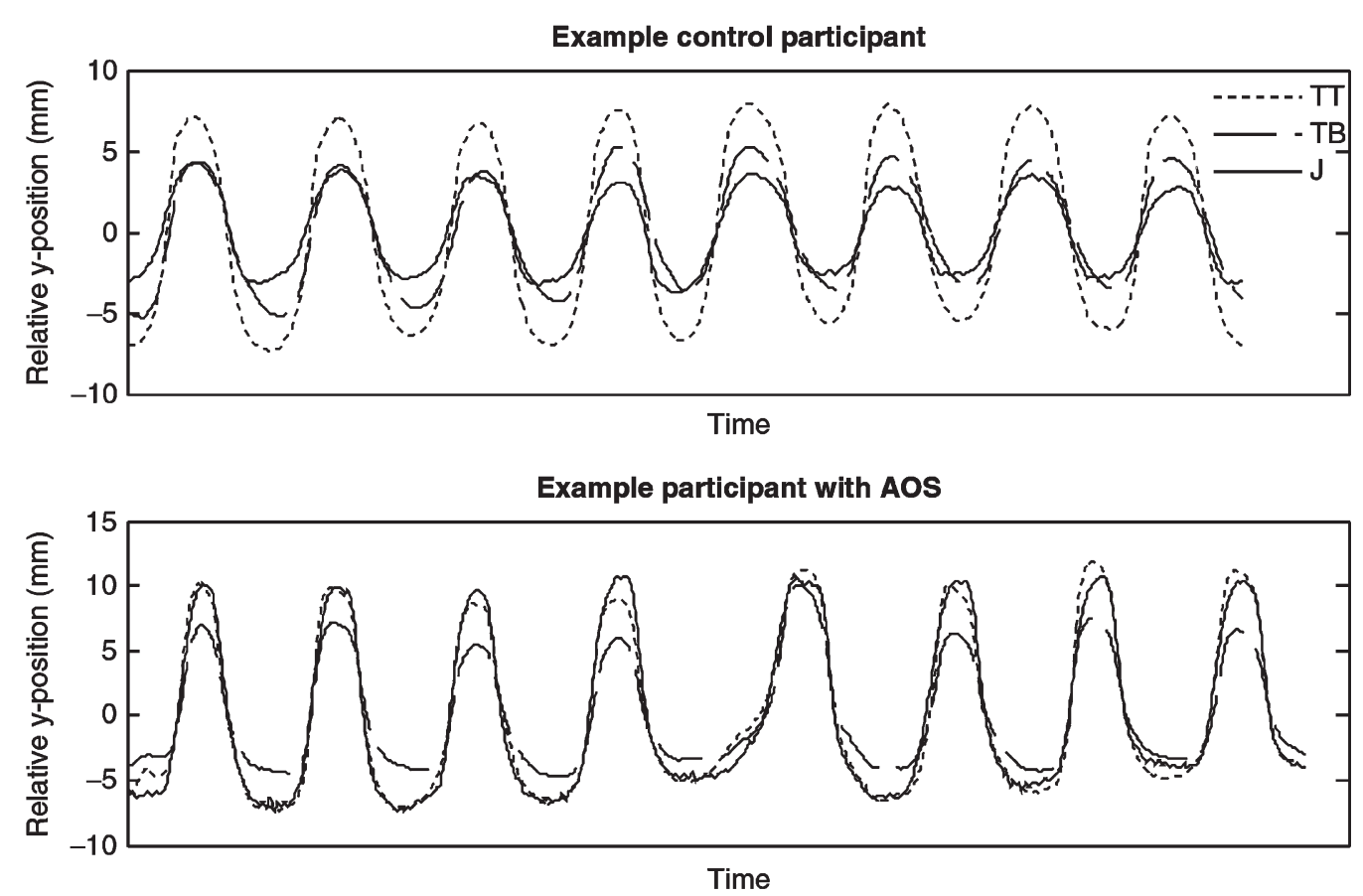

Figure 2. Relative tongue-tip (TT), tongue-back (TB) and jaw (J) movement during / ta/ syllable repetitions produced at a typical rate of speech, for an example control participant (top panel) and a participant with apraxia of speech (AOS; bottom panel).

the tongue. Thus, the purpose of the covariance analysis was to examine differences in articulatory coupling between the participants with AOS and healthy controls.

\section{Statistical analysis procedures}

Prior to statistical analysis, the kinematic data were screened for outliers. For the purpose of this study, an outlier was defined as a value that was greater than 3.29 $\mathrm{SD}$ above or below the group mean [35]. One outlier was identified and was replaced with the new group mean. Descriptive statistics were subsequently calculated for each target stimulus. For the control group, mean covariance values were derived from a maximum of 24 repetitions. Given the small participant numbers and the level of heterogeneity within the AOS group, each apraxic speaker's data was individually compared to the control group. The mean covariance values obtained by each of the participants with AOS were considered significantly different from the group of healthy talkers if they were greater than $2 \mathrm{SD}$ above or below the control group mean.

\section{Case reports}

Mean covariance values quantifying the strength of articulatory coupling are displayed in Figures 4-7. Comparisons were made between the typical rate produc- tions of the control group and the typical rate productions of each of the participants with AOS.

Figure 8 provides a visual representation of average rate of speech, expressed as syllables per second (SPS), for the control group and each of the participants with AOS for typical and fast rate conditions. On average, the control speakers spoke at 3.9 SPS $(\mathrm{SD}=0.12)$ when instructed to speak at a fast rate of speech and at 2.09 SPS $(S D=0.07)$ when instructed to speak at a typical rate of speech. In contrast, the participants with AOS, on average, spoke at 2.49 SPS $(\mathrm{SD}=0.68)$ when asked to speak at a fast of speech and at 1.42 SPS $(S D=0.42)$ when asked to speak at a typical rate of speech. Given that rate of speech was most comparable between the typical rate productions of the control group and the fast rate productions of the participants with AOS, additional comparisons of articulatory coupling were made between the typical rate productions of the control group and the fast rate productions of the participants with AOS.

The kinematic results obtained for each participant with AOS will be discussed in the following case discussions.

\section{AOS case 1 (A1)}

Analysis of A1's kinematic data revealed increased $\mathrm{TB} \times \mathrm{J}$ and $\mathrm{TT} \times \mathrm{TB}$ coupling during alveolar speech targets (i.e. /ta, sa/). A1 exhibited significantly greater $\mathrm{TB} \times \mathrm{J}$ coupling during typical rate $(M=1.08 \mathrm{SPS})$ pro- 

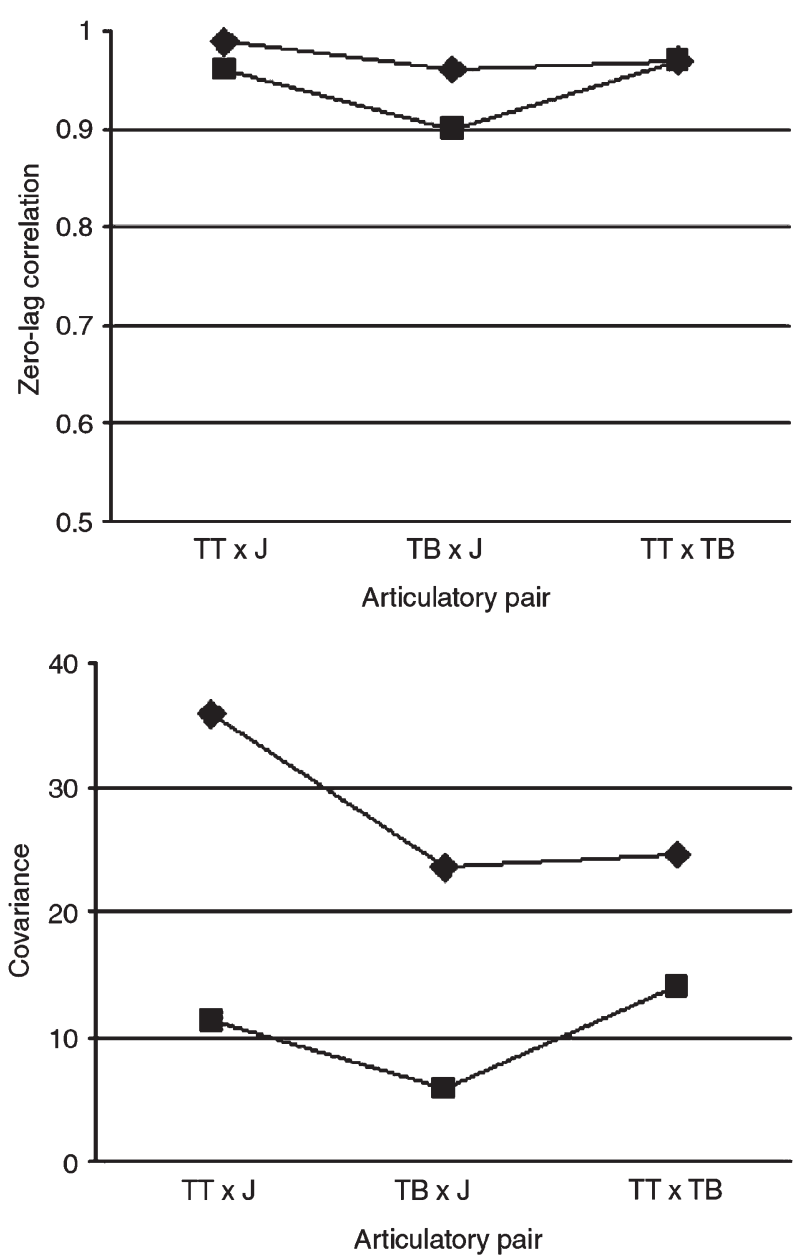

Figure 3. Top graph reflects the zero lag correlation values and the bottom graph reflects the resultant covariance values, for an example control participant (squares) and a participant with apraxia of speech (diamonds) during / ta/ syllable repetitions produced at a typical rate of speech. Strong spatiotemporal coupling/low degree of functional independence was inferred from a high covariance value.

ductions of $/ \mathrm{ta} /$ and fast rate $(M=2.45 \mathrm{SPS})$ productions of /ta/ and /sa/ (see Figures 4 and 5), relative to the control group. Significantly greater TT $\times$ TB coupling was evident during typical rate productions of / ta/ and fast rate repetitions of / sa/ (see Figures 4 and 5). With respect to the velar target $/ \mathrm{ka} /$, significantly greater lingual (i.e. TT $\times \mathrm{TB}$ ) coupling was observed during fast rate productions only, in comparison to the control group (see Figure 7). The strength of A1's TT $\times$ J coupling was comparable to the control group during typical and fast rate conditions of all target stimuli (see Figures 4-7).

Evidence of significantly greater articulatory coupling could be indicative of decreased functional movement independence. This generally appeared to be more apparent when A1 was instructed to speak at a fast speaking rate, which could indicate that, when A1's speech system was taxed, she lost her ability to exert in-

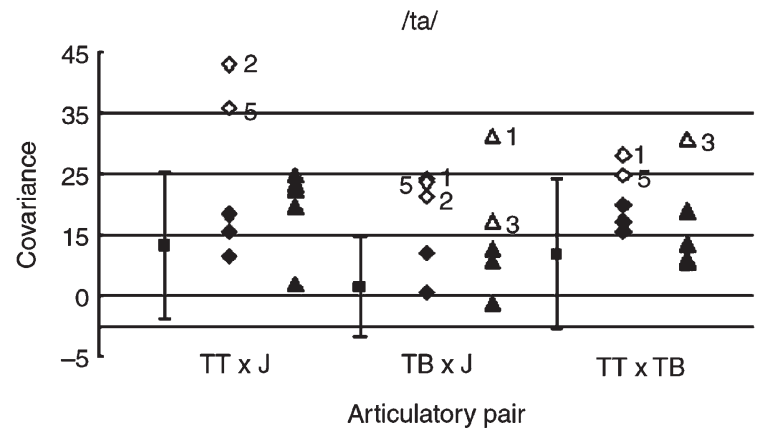

Figure 4. Average covariance values for the control group ( $n$ $=12$; typical rate $=$ squares) and each participant with apraxia of speech $(n=5$; typical rate = diamonds; fast rate $=$ triangles $)$ for / ta/ syllable repetitions, expressed as a function of articulatory pair. Error bars represent \pm 2 SDs. White diamonds/triangles represent statistically significant (i.e. $>2$ SDs above control group mean) values for $x$ participant. TT $=$ tongue-tip, TB = tongue-back, $\mathrm{J}=$ jaw.

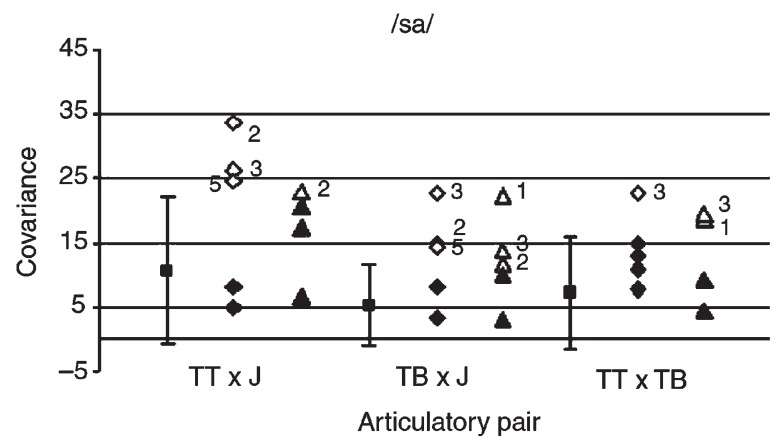

Figure 5. Average covariance values for the control group ( $n$ $=12$; typical rate $=$ squares) and each participant with apraxia of speech $(n=5$; typical rate $1 / 4$ diamonds; fast rate $=$ triangles $)$ for / sa/ syllable repetitions, expressed as a function of articulatory pair. Error bars represent \pm 2 SDs. White diamonds/triangles represent statistically significant (i.e >2 SDs above control group mean) values for $x$ participant. TT $=$ tongue-tip, TB = tongue-back, $\mathrm{J}=$ jaw.

dependent control over her articulators. On the other hand, she might have tried to simplify articulatory control by controlling the articulators as a single unit. Alternatively, given that covariance is sensitive to movement amplitude (i.e. covariance reflects the standard deviation of articulator movement, in addition to the zerolag correlation), the aforementioned observation could also imply that A1 relied on more expansive articulatory movement to generate a faster rate of speech. This would be considered an active strategy to increase syllable repetition rate that would require increased articulatory effort [28].

A1 appeared to exhibit significantly greater tongueback and jaw coupling, in the context of relatively comparable tongue-tip and jaw coupling to the control group, during alveolar speech targets. In contrast to the tongueback whose movement is constrained by connective 


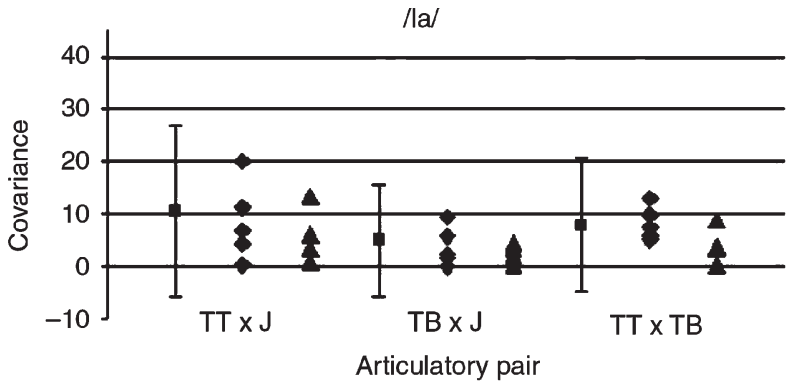

Figure 6. Average covariance values for the control group $(n$ $=12$; typical rate $=$ squares) and each participant with apraxia of speech $(n=5$; typical rate $=$ diamonds; fast rate $=$ triangles $)$ for /la/ syllable repetitions, expressed as a function of articulatory pair. Error bars represent $\pm 2 \mathrm{SDs}$. $\mathrm{TT}=$ tongue-tip, $\mathrm{TB}=$ tongue-back, J = jaw.

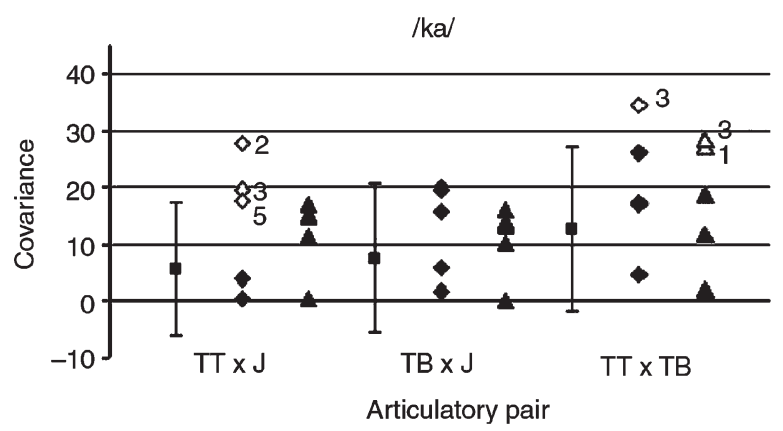

Figure 7. Average covariance values for the control group ( $n$ $=12$; typical rate $=$ squares) and each participant with apraxia of speech $(n=5$; typical rate $=$ diamonds; fast rate $=$ triangles $)$ for / ka/ syllable repetitions, expressed as a function of articulatory pair. Error bars represent \pm 2 SDs. White diamonds/triangles represent statistically significant (i.e. >2 SDs above control group mean) values for $x$ participant. TT $=$ tongue-tip, TB $=$ tongue-back, $\mathrm{J}=$ jaw.

tissue and extrinsic musculature [36], the tongue-tip has a large range of mobility that would presumably be more difficult to control. Despite this, A1 appeared to maintain adequate control over her tongue-tip and jaw coupling.

\section{AOS case 2 (A2)}

Significantly greater $\mathrm{TT} \times \mathrm{J}$ and $\mathrm{TB} \times \mathrm{J}$ coupling were recorded for A2 during alveolar (with the exception of $/ 1 /$ ) and velar speech targets, relative to the control group. With respect to $\mathrm{TT} \times \mathrm{J}$ coupling, significant differences were observed during typical rate $(M=1.82$ SPS) productions of / ta, sa, ka/ (see Figures 4, 5 and 7); however, these were only maintained during fast rate $(M=$ 2.57 SPS) productions of / sa/. Likewise, in comparison to the control group, significantly greater $\mathrm{TB} \times \mathrm{J}$ coupling was recorded during typical rate productions of / ta, sa/ and fast rate productions of /sa/ (see Figures 4 and 5).

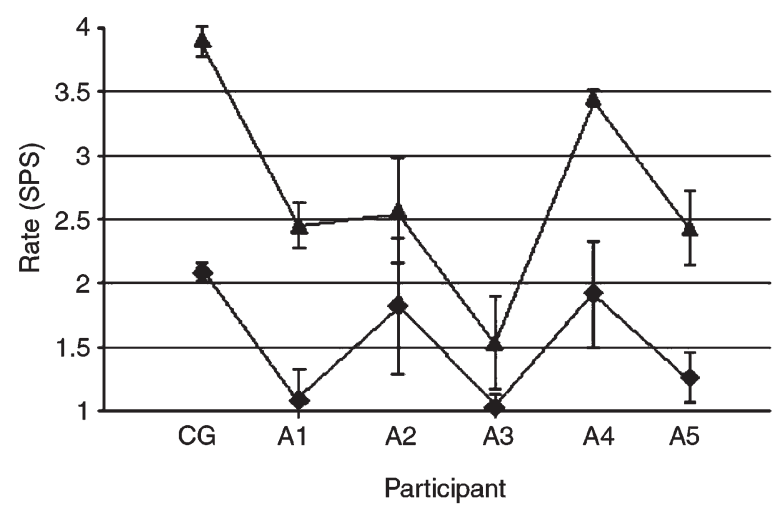

Figure 8. Average rate of speech for the control group (CG) $(n=12)$ and each participant with apraxia of speech (A1-A5) based on the syllable repetition task, expressed as syllables per second (SPS). Diamonds represent typical rate and triangles represent fast rate. Error bars represent $\pm 1 \mathrm{SD}$.

The strength of $\mathrm{A} 2$ 's $\mathrm{TB} \times \mathrm{J}$ coupling recorded during / $\mathrm{ka} /$ productions was comparable to the control group (see Figure 7). No significant differences in lingual coupling (i.e. TT $\times \mathrm{TB}$ ) were evident during typical and fast rate repetitions of / $\mathrm{ta}$, sa, la, ka/ (see Figures 4-7).

Thus, while A2 appeared able to exert independent control over her tongue-tip and tongueback, she demonstrated highly coupled tongue and jaw movements during alveolar and velar targets. It could be speculated that A2 was attempting to simplify articulatory control by controlling her tongue and jaw as a single unit. Significantly greater tongue-jaw coupling was also observed during / sa/ productions when A2 increased her syllable repetition rate, but, unlike A1, the strength of coupling decreased from the typical rate condition to the fast rate condition. This finding might indicate that $\mathrm{A} 2$ decreased the total distance travelled by her tongue to increase her speech rate. This would be considered a reactive strategy (i.e. requires less articulatory effort) and is comparable to that generally employed by healthy talkers $[27,28]$.

\section{AOS case 3 (A3)}

In comparison to the control group, $\mathrm{A} 3$ exhibited significantly greater $\mathrm{TT} \times \mathrm{J}, \mathrm{TB} \times \mathrm{J}$ and $\mathrm{TT} \times \mathrm{TB}$ coupling during alveolar (with the exception of $/ 1 /$ ) and velar speech targets. Significantly greater $\mathrm{TT} \times \mathrm{J}$ coupling was recorded during typical rate $(M=1.03 \mathrm{SPS})$ productions of / sa, ka/ (see Figures 5 and 7). Disturbances in TB $\times \mathrm{J}$ and TT $\times$ TB coupling were evident during typical $(M=$ 1.03 SPS) and fast ( $M=1.53 \mathrm{SPS})$ rate repetitions of $/ \mathrm{sa} /$ (see Figure 5), but were only apparent during fast rate ( $M=1.53$ SPS) repetitions of / ta/ (see Figure 4). Likewise, significantly greater lingual coupling (i.e. TT×TB) 
was observed during typical and fast rate productions of $/ \mathrm{ka} /$, relative to the control group (see Figure 7).

Overall, A3 exhibited tightly coupled articulatory movements during alveolar and velar target consonants, indicative of reduced functional movement independence between his tongue and jaw and tongue-tip and tongue-back. With the exception of $\mathrm{TT} \times \mathrm{J}$ coupling, these disturbances were maintained during fast rate repetitions; however, it should be highlighted that A3's fast rate of speech remained substantially slower than the average typical rate of speech produced by the control group. Nonetheless, it appeared sufficient to tax A3's speech system; articulatory movement was significantly more coupled for / ta/ during the fast rate condition only. Consistent with A1 and A2, the observation of increased articulatory coupling might indicate that A3 was trying to simplify articulatory control by reducing the total degrees of freedom within the articulatory system.

A3 appeared to be using two different strategies to increase his rate of speech. The strength of coupling was greatest during his fast rate productions of / ta/ and typical rate productions of $/ \mathrm{sa} /$ and $/ \mathrm{ka} /$. Thus, while A3 might have been able to generate sufficient speed during / sa/ and / $\mathrm{ka} /$ by reducing the total distance travelled by his tongue, he might have required more expansive tongue movement to generate faster articulatory speed during / ta/ repetitions.

\section{AOS case 4 (A4)}

Analysis of A4's kinematic data revealed comparable strengths of TT $\times \mathrm{J}, \mathrm{TB} \times \mathrm{J}$ and TT $\times \mathrm{TB}$ coupling to the control group during / $\mathrm{ta}, \mathrm{sa}, \mathrm{la}, \mathrm{ka} /$ syllable productions, produced at typical ( $M=1.92$ SPS) and fast $(M=3.45$ SPS) rates of speech (see Figures 4-7). Therefore, A4's kinematic results suggest that his ability to exert independent control over functionally-independent articulatory regions was preserved. It should be noted, however, that A4 did speak softly during the syllable repetition task, which might have impacted on his speech kinematics [37] and, subsequently, the strength of his articulatory coupling.

\section{AOS case 5 (A5)}

Significantly greater $\mathrm{TT} \times \mathrm{J}$ and $\mathrm{TB} \times \mathrm{J}$ coupling were recorded for A5 during alveolar (with the exception of $/ 1 /$ ) and velar target consonant productions, relative to the control group. TT $\times \mathrm{J}$ coupling was significantly stronger during typical rate ( $M=1.26 \mathrm{SPS})$ productions of / ta, sa, $\mathrm{ka} /$, as was $\mathrm{TB} \times \mathrm{J}$ coupling during typical rate productions of / ta, sa/ (see Figures 4, 5 and 7). TT $\times$ TB cou- pling was only significant, relative to the control group, during typical rate productions of /ta/ (see Figure 4). No significant differences in articulatory coupling were recorded during fast rate ( $M=2.43$ SPS) productions of /ta, sa, la, ka/ (see Figures 4-7).

Thus, similar with the initial three cases discussed, A5 appeared to be attempting to simplify articulatory control by controlling the tongue and jaw as a single unit during alveolar and velar consonants. In general, however, A5's ability to exert independent control over her tongue tip and tongueback appeared to be preserved. Interestingly, the strength of coupling did not remain significant when A5 increased her rate of speech.

\section{General discussion}

Overall, the participants with AOS appeared to rely on increased articulatory coupling during alveolar and velar target consonant productions, which is consistent with the hypothesis that persons with AOS would exhibit decreased functional movement independence across different articulatory regions. Similarly, an increase in speech rate typically resulted in weaker articulatory coupling. However, A1 and A3 both exhibited instances of stronger coupling during the fast rate condition. Accordingly, there were many occasions where the strength of articulatory coupling, in particular, between the tongue-back and jaw and the tonguetip and tongueback, was significantly greater for the participants with AOS, relative to the control group, during fast rate productions of / ta, sa, ka/. Analysis of the kinematic properties underlying increased articulatory coupling was, however, beyond the realm of the present study and therefore the conclusions drawn in the following discussion are speculative.

A reduction in functional movement independence might have reflected an attempt to simplify articulatory control. To promote efficiency within complex, dynamical systems (e.g. speech system), individual components (e.g. articulators) typically combine to form functional synergies (e.g. coordinative structures), which in turn reduce the total degrees of freedom made available to the system $[38,39]$. Given that the strength of articulatory coupling exhibited by the participants with AOS was, in general, significantly greater than that recorded for the control group, it could be postulated that the participants with AOS tried to enforce a stronger link between articulators to further reduce the total degrees of freedom needed to be controlled. In this case, the increase in articulatory coupling might have reflected a control strategy for coping with an impaired articulatory system. 
Alternatively, increased articulatory coupling might have reflected a decrease in neural control over distinct articulators. Highly synchronous articulatory movement, like that recorded in the current study, is often observed in children [40-43]. Therefore, the decrease in functional movement independence might suggest that some speakers who present with AOS lose the ability to exert independent control over quasi-independent articulatory structures. The tongue tip/blade and tongue body, in particular, have been found to function relatively independently of each other in a mature speech system [40,41]. Three of the five participants with AOS exhibited significantly greater lingual coupling during single speech gestures, relative to the control group. This result is consistent with the large body of research that has identified double alveolar-velar contact patterns in participants with AOS [21-26]. Poorly differentiated lingual movement could account, in part, for the sound distortions exhibited by the participants with AOS in the present study.

Given that covariance is sensitive to movement amplitude, it could also be speculated that the increase in coupling reflected an increase in distance travelled by the tongue and/or jaw. A reduction in movement amplitude has been found to have a destabilizing effect on the speech mechanism $[28,44,45]$ and therefore the participants with AOS might have employed larger articulatory movements in an effort to increase tongue and/or jaw stability, at the risk of being less efficient.

An increase or decrease in articulatory coupling from the typical rate condition to the fast rate condition might also reflect an increase or decrease in the total distance travelled by the tongue. Indeed, Hertrich and Ackermann [29] identified that the constrictor articulators were more sensitive than the jaw to changes in speech rate. Neurologically unimpaired speakers have been found to employ a variety of strategies to increase syllable repetition rate, one being a reactive strategy wherein they reduce the total distance travelled by the tongue in an attempt to economize effort $[27,28]$. The participants with AOS who produced a decrease in articulatory coupling in the fast rate condition might therefore have been employing a similar strategy to neurologically sound participants. In contrast, participants who produced stronger articulatory coupling during the fast rate condition might have been employing an active strategy; they might have relied on more expansive lingual movement to generate faster speeds (i.e. increased velocity). Alternatively, the increase or decrease in articulatory coupling might simply reflect the impact of speech rate on articulatory control.
Interestingly, negative coupling between the tonguetip and tongue-back, like that reported by Green and Wang [20] during alveolar sound production, was not recorded in the present study. The most likely reason for this discrepancy is that, in the current study, tongue movement was not expressed independently from jaw movement. Consequently, the covariance values in this study were biased toward high, positive coupling between the tongue-tip and tongue-back. Another reason for this discrepancy could be receiver coil placement. The tongue-back coil in the current study was placed at $4 \mathrm{~cm}$ from the tongue-tip, which is considered to be the tongue-body, not tonguedorsum. In Green and Wang's [20] study, the tongue-tip and tongue-body were negatively coupled during alveolar sound production, but to a substantially lesser extent, relative to tongue-tip and tonguedorsum coupling (i.e. $\mathrm{Cov}=-0.17$ and -3.37 , respectively).

\section{Conclusion}

Overall, the decrease in functional movement independence recorded by four participants with AOS in the present study might have reflected an attempt to reduce the total degrees of freedom within the articulatory system, to ultimately simplify articulatory control; or, alternatively, a more primitive level of neural control. On the other hand, it might have reflected an increase in tongue and/or jaw amplitude. Interestingly, evidence for reduced functional movement independence was not confined to the stimuli produced at a slower rate of speech.

The preceding discussion was designed to provide an overview of the general trends alluded to in the individual case discussions; however, it should be reiterated that the participants with AOS each presented with different movement profiles. For example, A4 appeared to maintain functional movement independence across quasi-independent articulatory structures, whereas the other four participants with AOS involved in the study appeared unable to. It is important that the trends outlined above are not generalized to all patients presenting with AOS; rather, that they help inform as to what might underlie the articulatory disturbances the client presents with. One reason for the inter-participant variability revealed in the current study could be the presence of different degrees of AOS. A1 was diagnosed with a moderate-severe AOS, whereas A5, for example, was diagnosed with a mild AOS. Additionally, AOS, by nature, is characterized by a high degree of inter-participant variability $[1,3]$. 


\section{Methodological limitations and future directions}

It is important that the present research findings are interpreted in the context of the following methodological limitations. First, each of the participants with AOS presented with a concomitant non-fluent aphasia; the presence of aphasia was difficult to control for as few patients present with pure AOS [1]. Given that the EMA assessment involved a syllable repetition task, it is unlikely that the presence of a concomitant non-fluent aphasia would have impacted on the results. Nevertheless, the aforementioned research findings do need to be interpreted with caution as a control group of participants with a non-fluent aphasia (in the absence of AOS) was not included in the study to verify this assumption. Secondly, the conclusions drawn in the discussion were speculative as the kinematic properties underlying the increase in coupling were not examined. Accordingly, investigation of isolated tongue and jaw movements and tongue-to-palate contacts using EMA and EPG methodology, respectively, would complement the current results and improve one's understanding of the underlying nature of AOS.

Despite the aforementioned limitations, the present study emphasizes the advantages of using physiologic instrumentation to determine the underlying nature of articulatory errors (e.g. sound distortions) in individuals with AOS. In the context of a high degree of intra-participant variability, however, it is important that each apraxic speaker's speech be examined individually using EMA prior to treatment planning. The present results are preliminary, given that they are based on a small sample of five participants with AOS, but do indicate that treatment aimed at increasing functional movement independence between articulators could assist in improving articulation in patients with AOS. Biofeedback therapy, using EMA and/or EPG, could prove beneficial in achieving this treatment goal.

\section{Acknowledgments}

The authors acknowledge the valuable assistance of the Princess Alexandra Hospital, The Prince Charles Hospital, RSL Care and the Acacia Ridge Stroke Support Group, Queensland, in participant recruitment and would like to extend their thanks to the participants for their involvement in the research project. The first author was a recipient of a University of Queensland Graduate School Research Travel Grant.

\section{References}

1. Duffy JR. Motor speech disorders: Substrates, differential diagnosis, and management. 2nd ed. Philadelphia, PA: Mosby; 2005.

2. McNeil M, Pratt SR, Fossett TRD. The differential diagnosis of apraxia of speech. In: Maassen B, Kent R, Peters H, van Lieshout $\mathrm{P}$, Hulstijn $\mathrm{W}$, editors. Speech motor control in normal and disordered speech. Oxford: Oxford University Press; 2004. pp 389-413.

3. McNeil M, Robin DA, Schmidt RA. Apraxia of speech: Definition, differentiation, and treatment. In: McNeil MR, editor. Clinical management of sensorimotor speech disorders. New York: Thieme; 1997. pp 311-344.

4. Odell KH, McNeil M, Rosenbek JC, Hunter L. Perceptual characteristics of consonant production by apraxic speakers. Journal of Speech and Hearing Disorders 1990;55:345-359.

5. Odell KH, McNeil M, Rosenbek JC, Hunter L. Perceptual characteristics of vowel and prosody production in apraxic, aphasic, and dysarthric speakers. Journal of Speech and Hearing Research 1991;34:67-80.

6. Bose A, van Lieshout P, Square PA. Kinematic characteristics of bilabial closure in an individual with Broca's aphasia and apraxia of speech. Paper presented at the $4^{\text {th }}$ International Speech Motor Conference; 13-16 June 2001; Nijmegen.

7. McNeil M, Adams S. A comparison of speech kinematics among apraxic, conduction aphasic, ataxic dysarthric, and normal geriatric speakers. Clinical Aphasiology 1991;19: 279-294.

8. McNeil M, Caligiuri MP, Rosenbek JC. A comparison of labiomandibular kinematic durations, displacements, velocities, and dysmetrias in apraxic and normal adults. Clinical Aphasiology 1989;18:173-193.

9. Itoh M, Sasanuma S, Hirose H, Yoshioka H, Ushijima T. Abnormal articulatory dynamics in a patient with apraxia of speech: X-ray microbeam observation. Brain and Language 1980;11:66-75.

10. Mauszycki SC, Dromey C, Wambaugh JL. Variability in apraxia of speech: A perceptual, acoustic, and kinematic analysis of stop consonants. Journal of Medical SpeechLanguage Pathology 2007;15:223-242.

11. Robin DA, Bean C, Folkins JW. Lip movement in apraxia of speech. Journal of Speech and Hearing Research 1989;32: 512-523.

12. Bartle CJ, Goozée JV, Murdoch BE. An EMA analysis of the effect of increasing word length on consonant production in apraxia of speech: A case study. Clinical Linguistics and Phonetics 2007;21:189-210.

13. Bartle-Meyer CJ, Goozée JV, Murdoch BE. Kinematic analysis of consonant production in acquired apraxia of speech. Journal of Medical Speech, Lanugage Pathology; in press.

14. Bartle-Meyer CJ, Goozée JV, Murdoch BE. Kinematic investigation of lingual movement in words of increasing length in acquired apraxia of speech. Clinical Linguistics and Phonetics; in press. 
15. Saltzman E, Munhall K. A dynamical approach to gestural patterning in speech production. Ecological Psychology 1989;1:333-382.

16. Gracco VL, Abbs JH. Variant and invariant characteristics of speech movements. Experimental Brain Research 1986;65: 156-166.

17. Mooshammer C, Geumann A, Hoole P, Alfonso P, van Lieshout P, Fuchs $S$. Coordination of lingual and mandibular gestures for different manners of articulation. $15^{\text {th }}$ International Congress of Phonetic Sciences; 2003; Barcelona. $81 \mathrm{p}$.

18. Mooshammer C, Hoole P, Geumann A. Interarticulator cohesion within coronal consonant production. Journal of the Acoustical Society of America 2006;120:1028-1039.

19. Mooshammer C, Hoole P, Geumann A. Jaw and order. Language and Speech 2007;50:145-176.

20. Green JR, Wang YT. Tongue-surface movement patterns during speech and swallowing. Journal of the Acoustical Society of America 2003;113:2820-2833.

21. Hardcastle WJ, Edwards S. EPG-based description of apraxic speech errors. In: Kent RD, editor. Intelligibility in speech disorders: Theory, measurement and management. Amsterdam: John Benjamins Publishing; 1992. pp 287-328.

22. Hardcastle WJ. Profiling lingual-palatal contact patterns in normal and dyspraxic speech. In: Clark JE, editor. The cultivated Australian: Festschrift in honour of Arthur Delbridge. Hamburg: H. Buske; 1985. pp 417-427.

23. Hardcastle WJ. Electropalatographic study of articulation disorders in verbal dyspraxia. In: Ryalls JH, editor. Phonetic approaches to speech production in aphasia and related disorders. Boston: Little, Brown, and Company; 1987. pp 113-136.

24. Hardcastle WJ, Morgan Barry RA, Clark CJ. Articulatory and voicing characteristics of adult dysarthria and verbal dyspraxic speakers: An instrumental study. British Journal of Disorders of Communication 1985;20:249-270.

25. Ingram J, Hardcastle W. Perceptual, acoustic and electropalatographic evaluation of coarticulation effects in apraxic speech. In: Seidl R, editor Proceedings of the Third Australian International Conference on Speech Science and Technology. Canberra: Australian Speech Science and Technology Association; 1990. pp. 110-115.

26. Wood S. Electropalatographic study of speech sound errors in adults with acquired aphasia [dissertation]. Edinburgh: Queen Margaret College; 1997.

27. Goozée JV, LaPointe LL, Murdoch BE. Effects of speaking rate on EMA-derived lingual kinematics: A preliminary investigation. Clinical Linguistics \& Phonetics 2003;17:375-381.

28. Goozée JV, Stephenson DK, Murdoch BE, Darnell RE, LaPointe LL. Lingual kinematic strategies used to increase speech rate: Comparison between younger and older adults. Clinical Linguistics \& Phonetics 2005;19:319-334.
29. Hertrich I, Ackermann H. Lip-jaw and tongue-jaw coordination during rate-controlled syllable repetitions. Journal of the Acoustical Society of America 2000;107:2236-2247.

30. Dabul B. Apraxia battery for adults. 2nd ed. Austin, TX: Proed; 2000.

31. Goodglass H. The Boston diagnostic aphasia examination. Philadelphia: Lippincott Williams \& Wilkins; 2001.

32. Enderby P. Frenchay dysarthria assessment. San Diego: College Hill Press; 1983.

33. Darley FL, Aronson AE, Brown JR. Motor speech disorders. Philadelphia, PA: W.B. Saunders; 1975.

34. Goozée JV, Murdoch BE, Theodoros DG, Stokes PD. Kinematic analysis of tongue movements in dysarthria following traumatic brain injury using electromagnetic articulography. Brain Injury 2000;14:153-174.

35. Field A. Discovering statistics using SPSS. 2nd ed. London: Sage Publications; 2005.

36. Wilson E, Green J. Coordinative organization of lingual propulsion during the normal adult swallow. Dysphagia 2006;21:226-236.

37. Tasko SM, McClean MD. Variations in articulatory movement with changes in speech task. Journal of Speech, Language, and Hearing Research 2004;47: 85-100.

38. Kelso JAS. Dynamic patterns: The self-organization of brain and behavior. Cambridge: MIT Press; 1995.

39. Kelso JAS. Principles of dynamic pattern formation and change for a science of human behavior. In: Bergman LR, Cairns RB, Nilsson LG, Nystedt L, editors. Developmental science and the holistic approach. Mahwah, NJ: Lawrence Erlbaum Associates; 2000. pp 63-83.

40. Cheng HY, Murdoch BE, Goozée JV, Scott D. Electropalatographic assessment of tongue-to-palate contact patterns and variability in children, adolescents, and adults. Journal of Speech, Language, and Hearing Research 2007;50:375-392.

41. Gibbon FE. Undifferentiated lingual gestures in children with articulation/phonological disorders. Journal of Speech, Language, and Hearing Research 1999;42:382-397.

42. Green JR, Moore CA, Higashikawa M, Steeve RW. The physiologic development of speech motor control: Lip and jaw coordination. Journal of Speech, Language, and Hearing Research 2000;43:239-255.

43. Kent RD. The segmental organization of speech. In: MacNeilage PF, editor. The production of speech. New York: Springer-Verlag; 1983. pp 56-89.

44. Van Lieshout PHHM, Rutjens CAW, Spauwen PHM. The dynamics of interlip coupling in speakers with a repaired unilateral cleft-lip history. Journal of Speech, Language, and Hearing Research 2002;45:5-19.

45. Van Lieshout PHHM, Bose A, Square PA, Steele CM. Speech motor control in fluent and dysfluent speech production of an individual with apraxia of speech and Broca's aphasia. Clinical Linguistics \& Phonetics 2007;21: 159-188. 